\title{
MONOIDS OF ND-FULL HYPERSUBSTITUTIONS
}

\author{
SOMSAK LEKKOKSUNG \\ Division of Mathematics, Faculty of Engineering \\ Rajamangala University of Technology Isan \\ Khon Kaen Campus, Khon Kaen 40000, Thailand \\ e-mail: lekkoksung_somsak@hotmail.com
}

\begin{abstract}
An nd-full hypersubstitution maps any operation symbols to the set of full terms of type $\tau_{n}$. Nd-full hypersubstitutions can be extended to mappings which map sets of full terms to sets of full terms. The aims of this paper are to show that the extension of an nd-full hypersubstitution is an endomorphism of some clone and that the set of all nd-full hypersubstitutions forms a monoid.
\end{abstract}

Keywords: superposition of sets of terms, clone, hypersubstitution.

2010 Mathematics Subject Classification: 08C99, 68Q70.

\section{REFERENCES}

[1] Th. Changphas and K. Denecke, Full hypersubstitutions and full solid varieties of semigroups, East-West J. Math. 4 (2002) 177-193.

[2] K. Denecke, J. Koppitz and SI. Shtrakov, The depth of a hypersubstitution, J. Automata, Languages and Combin. 6,3 (2001) 253-262.

[3] K. Denecke and P. Jampachon, Clones of full terms, Algebra Discrete Math. 4 (2004) $1-11$.

[4] K. Denecke, P. Glubudom and J. Koppitz, Power clones and non-deterministic hypersubstitutions, Asian-European J. Math. 1,2 (2008) 177-188.

doi:10.1142/S1793557108000175

[5] E. Graczyńska and D. Schweigert, Hypervarieties of a given type, Algebra Universalis 27 (1990) 305-318.

doi:10.1007/BF01190711

Received 28 November 2018

Revised 5 March 2019

Accepted 13 June 2019 